\title{
MODULUS OF CURVE FAMILIES AND EXTREMALITY OF SPIRAL-STRETCH MAPS
}

\author{
By \\ Zoltán M. Balogh, Katrin Fässler, and IoAnnis D. Platis*
}

\begin{abstract}
We develop a method using the modulus of curve families to study minimisation problems for the mean distortion functional in the class of finite distortion homeomorphisms. We apply our method to prove extremality of the spiral-stretch mappings defined on annuli in the complex plane. This generalises results of Gutlyanskiı̌ and Martio [12] and Strebel [23].
\end{abstract}

\section{Introduction}

The study of extremal problems of mappings of finite distortion was initiated by Astala, Iwaniec, Martin, and Onninen in [5]. These mappings are generalisations of quasiconformal mappings without uniform bound on their distortion. Whereas for quasiconformal mappings one is usually interested in minimisers of the maximal distortion, for mappings of finite distortion a mean distortion functional is to be minimised. This viewpoint was clearly indicated in the recent monograph [4]. From the discussion in [4], it becomes evident that the problem of minimising the mean distortion is different from the one for the maximal distortion and new techniques are needed. For example, Martin showed in [18] that, in contrast to the classical case, the Teichmüller problem for the mean distortion does not have a solution in the class of mappings with finite distortion.

In the present paper, we pursue another approach, complementing the techniques in $[4,5]$, to identify the extremal mapping within a given class of mappings of finite distortion. This procedure uses modulus estimates for curve families. The modulus of a curve family is a geometric quantity which is quasi-invariant under quasiconformal mappings and therefore yields a lower bound on the maximal distortion for an appropriate choice of curve family. Similarly, it was shown in [20] that the technique of modulus estimation can be used for mappings of finite

\footnotetext{
${ }^{*}$ The authors were supported by Swiss National Science Foundation, European Research Council Project GALA and European Science Foundation Project HCAA
} 
distortion. We use this approach also in our work as a general tool to treat the minimisation problem for a weighted mean distortion with fixed boundary data.

We illustrate how to apply this general method to mappings of finite distortion on the annulus with boundary values given by a composition of a logarithmic spiral and radial stretching map. More precisely, we look at the following problem.

Consider "all" self-maps of an annulus in the plane that leave the outer boundary pointwise fixed and rotate the inner boundary by a given angle. Identify a mapping in this class that is "as conformal as possible."

This problem was studied first for the class of quasiconformal mappings, where a natural way to describe how close such a mapping is to a conformal map is to consider its maximal distortion. Strebel proved in [23] that the logarithmic spiral map is a solution of the above problem in this class; that is, it is a minimiser for the maximal distortion. More recently, Gutlyanskiı and Martio [12] have shown that it is also a minimiser for a weighted mean distortion in the same class. This leads to estimates for the rotation angle and a sharp solution to John's angle distortion problem [14]. For a survey on results related to the logarithmic spiral map and its importance in applications, see [12].

One of the main results in the present paper is the solution of the above problem in the more general setting of mappings with finite distortion and also for mappings between two annuli of different radii. This boundary value minimisation problem has a $\mathfrak{C}^{\infty}$-smooth solution: the spiral-stretch map — the spiral map composed with the well-known radial stretching. Unlike in the quasiconformal case, the existence of an extremal map of finite distortion cannot be a priori guaranteed by the standard normal family argument. Because of the lack of this type of convergence, results obtained by the curve families technique are used. The curve family we employ extensively in this paper is a family of spirals which was used recently by Brakalova $[7,8]$ in the characterisation of conformality at a point.

As a second application of the modulus method, we prove the extremality of the radial-stretch map among all finite distortion homeomorphisms between two annuli that preserve inner and outer boundary. Related results have been obtained recently in [3] by invariant integral methods.

The paper is organised as follows. In Section 2, we provide the necessary definitions and notations and state our main results. The proofs are given in Sections 3 and 4. In addition, we discuss an example that illustrates the different nature of the minimisation problem for the maximal and the mean distortion in terms of the uniqueness of the extremal mapping. The last section is devoted to additional remarks and comments on related results. 


\section{Preliminaries and statements of the main results}

\subsection{Quasiconformal mappings and mappings of finite distortion.}

Let $\Omega$ and $\Omega^{\prime}$ be domains in $\mathbb{C}$ and consider a sense-preserving homeomorphism $f: \Omega \rightarrow \Omega^{\prime}$ of class $W_{l o c}^{1,2}(\Omega)$. Assume that there exists a measurable function $z \mapsto K(z)$, finite a.e., with

$$
|D f(z)|^{2} \leq K(z) J(z, f) \text { a.e., }
$$

where $|D f(z)|=\max \{|D f(z) v|:|v|=1\}$ is the norm of the formal differential $D f(z)$ and $J(z, f)=\operatorname{det} D f(z)$ is its Jacobian determinant. The map is called quasiconformal if there exists a constant $K \geq 1$ such that $K(z) \leq K$ holds almost everywhere.

We are interested in the larger class of mappings of finite distortion; see, e.g., $[15,13,16]$. Unlike quasiconformal maps, these mappings are not required to be homeomorphisms and there need be no uniform bound on $K(z)$. We consider only mappings of finite distortion that are homeomorphisms.

Definition 1. A function $f: \Omega \rightarrow \mathbb{C}$ on a domain $\Omega \subset \mathbb{C}$ is called a mapping of finite distortion if

(i) $f \in W_{\text {loc }}^{1,1}(\Omega, \mathbb{C})$;

(ii) $J(z, f)$ is locally integrable; and

(iii) there exists a measurable function $z \mapsto K(z)$, finite a.e., with

$$
|D f(z)|^{2} \leq K(z) J(z, f) \quad \text { a.e. }
$$

The (linear) distortion function is defined as

$$
K(z, f):=\left\{\begin{array}{ll}
\frac{|D f(z)|^{2}}{J(z, f)} & \text { if } J(z, f)>0 \\
1 & \text { otherwise }
\end{array}= \begin{cases}\frac{\left|f_{z}(z)\right|+\left|f_{\bar{z}}(z)\right|}{\left|f_{z}(z)\right|-\left|f_{\bar{z}}(z)\right|} & \text { if } J(z, f)>0 \\
1 & \text { otherwise. }\end{cases}\right.
$$

The maximal distortion is given by

$$
K_{f}:=\operatorname{ess}_{\sup _{z \in \Omega}} K(z, f),
$$

which is always finite for quasiconformal maps but may be infinite for mappings of finite distortion.

The minimisation of $K_{f}$ in a given class of quasiconformal mappings is a classical problem; the finite distortion class arises naturally while minimising the mean distortion functional

$$
f \mapsto \int_{\Omega} \Psi(K(z, f)) \rho^{2}(z) \mathrm{d} \mathcal{L}^{2}(z)
$$

where $\rho$ is a suitable density and $\Psi$ a continuous, convex, non-decreasing function on $[1, \infty)$ with $\Psi(1)=1$. 
2.2 Main results. We concentrate on the minimisation problem for the above functional in the class of mappings between annuli with certain boundary conditions. This is in some sense the first non-trivial case for which the extremal problem has an interesting solution. Recall that in the case of bounded simply connected domains without boundary data, the existence of a conformal solution is always guaranteed by the Riemann Mapping Theorem.

For $0<a<b$, we denote the annulus with centre at the origin, inner radius $a$ and outer radius $b$ by

$$
A(a, b):=\{z \in \mathbb{C}: a<|z|<b\} .
$$

We are considering mappings between two annuli of identical outer radius. For simplicity we may assume that the mappings are defined on annulus $A(q, 1)$, $0<q<1$.

We consider the following class of mappings.

Definition 2. Assume that $q \in(0,1), \theta \in[-\pi, \pi]$ and $k_{1}>0$ are arbitrary constants. We denote by $\mathcal{F}$ the class of $W_{\text {loc }}^{1,2}$-homeomorphisms of finite distortion

$$
f: A(q, 1) \rightarrow A\left(q^{k_{1}}, 1\right)
$$

with integrable distortion function $z \mapsto K(z, f)$ and extension to the boundary

$$
f(z)= \begin{cases}z, & |z|=1, \\ q^{k_{1}-1} z e^{\mathrm{i} \theta}, & |z|=q .\end{cases}
$$

Remark 3. Note that we have restricted the class $\mathcal{F}$ under consideration to the subclass comprising mappings of finite distortion that are also sense-preserving $W_{l o c}^{1,2}$-homeomorphisms with integrable distortion. These additional regularity assumptions are needed to apply the modulus estimate from Theorem 9 on which our proof is based. The conditions are known to be the optimal assumptions to ensure that

(1) $J(\cdot, f)>0$ a.e.; and

(2) $f$ satisfies Lusin's condition $(\mathrm{N})$.

There does not exist a conformal mapping in the class $\mathcal{F}$ unless $\theta=0$ and $k_{1}=1$. Still, one can identify a mapping that is as conformal as possible in the sense that it minimises a weighted mean distortion. Candidates for a solution of this extremal problem are the spiral-stretch maps $f_{N} \in \mathcal{F}, N \in \mathbb{Z}$, which are defined as follows. 
Definition 4. Let $N \in \mathbb{Z}$. The $\mathbf{N}$-th spiral-stretch map $f_{N}$ is defined by

$$
f_{N}(z):=z|z|^{k_{1}-1} e^{\mathrm{i} k_{2} \log |z|}, \quad z \in A(q, 1),
$$

where

$$
k_{2}:=k_{2}(N):=\frac{\theta+2 \pi N}{\log q} .
$$

We obtain the minimising property of $f_{N}$ in the subclass $\mathcal{F}_{N} \subset \mathcal{F}$ of mappings in $\mathcal{F}$ that are homotopic to $f_{N}$ with respect to the boundary.

Theorem 5. Let $q \in(0,1), \theta \in[-\pi, \pi]$ and $k_{1}>0$. For arbitrary $N \in \mathbb{Z}$,

$$
\int_{A(q, 1)} K\left(z, f_{N}\right) \frac{1}{|z|^{2}} \mathrm{~d} \mathcal{L}^{2}(z) \leq \int_{A(q, 1)} K(z, f) \frac{1}{|z|^{2}} \mathrm{~d} \mathcal{L}^{2}(z) \quad \text { for all } f \in \mathcal{F}_{N} .
$$

For each mapping $f$ in the class $\mathcal{F}$, an integer $N \in \mathbb{Z}$ can be found such that $f$ is homotopic to $f_{N}$. According to Theorem 5, the map $f_{N}$ is extremal in its homotopy class. Moreover, we show that $f_{0}$ is extremal among all $f_{N}, N \in \mathbb{Z}$. An application of Jensen's inequality then yields the following main result.

Theorem 6. Let $q \in(0,1), \theta \in[-\pi, \pi], k_{1}>0$. Then for every continuous, non-decreasing, convex function $\Psi:[1, \infty) \rightarrow[1, \infty)$ with $\Psi(1)=1$,

$$
\int_{A(q, 1)} \Psi\left(K\left(z, f_{0}\right)\right) \frac{1}{|z|^{2}} \mathrm{~d} \mathcal{L}^{2}(z) \leq \int_{A(q, 1)} \Psi(K(z, f)) \frac{1}{|z|^{2}} \mathrm{~d} \mathcal{L}^{2}(z) \quad \text { for all } f \in \mathcal{F},
$$

where $f_{0}(z)=z|z|^{k_{1}-1} e^{\mathrm{i} \frac{\theta}{\log q} \log |z|}$.

Theorem 6 generalises a result of Gutlyanski1̌ and Martio [12], who considered the analogous problem for quasiconformal self-maps of the annulus $A(q, 1)$. Indeed, by setting $k_{1}=1$, we obtain, as a Corollary to Theorem 6 , the extremality of the logarithmic spiral map $f_{0}(z)=z e^{i k_{2} \log |z|}, k_{2}=\theta / \log q$, in the smaller class of quasiconformal self-maps of $A(q, 1)$ that rotate the inner boundary by $\theta$ and leave the outer boundary pointwise fixed.

In the case $\theta=0$, the extremal map is the radial stretching $f_{0}(z)=z|z|^{k_{1}-1}$. This map plays a particular role in the theory of quasiconformal mappings, as it is the conjectured extremal mapping in Gehring's problem (solved by Astala [2] in the planar case) of higher integrability of the Jacobian [11].

An application of Theorem 6 to the case $\theta=0$ shows that the radial stretching minimises the mean distortion in the respective class of mappings from $A(q, 1)$ to $A\left(q^{k_{1}}, 1\right)$ (without rotation of the inner boundary). However, the same map turns out to be extremal for the mean distortion even in the larger class of finite distortion maps $A(q, 1) \rightarrow A\left(q^{k_{1}}, 1\right)$ that send the inner and outer boundary of $A(q, 1)$ to the inner and outer boundary of $A\left(q^{k_{1}}, 1\right)$, respectively, but do not necessarily leave the boundary pointwise fixed; see Theorem 8 below. 
Definition 7. Assume that $q \in(0,1)$ and $k_{1}>0$ are arbitrary constants. We define the class $\mathcal{G}$ to be the class of $W_{l o c}^{1,2}$-homeomorphisms of finite distortion

$$
f: A(q, 1) \rightarrow A\left(q^{k_{1}}, 1\right)
$$

with integrable distortion function $z \mapsto K(z, f)$ and extension to the boundary

$$
|f(z)|=q^{k_{1}} \text { for }|z|=q \text { and }|f(z)|=1 \text { for }|z|=1 .
$$

Theorem 8. Let $q \in(0,1)$ and $k_{1}>0$. Then for every continuous, nondecreasing, convex function $\Psi:[1, \infty) \rightarrow[1, \infty)$ with $\Psi(1)=1$,

$$
\int_{A(q, 1)} \Psi\left(K\left(z, f_{0}\right)\right) \frac{1}{|z|^{2}} \mathrm{~d} \mathcal{L}^{2}(z) \leq \int_{A(q, 1)} \Psi(K(z, f)) \frac{1}{|z|^{2}} \mathrm{~d} \mathcal{L}^{2}(z) \quad \text { for all } f \in \mathcal{G},
$$

where $f_{0}(z)=z|z|^{k_{1}-1}$.

A similar result was obtained in [3] for the case of the non-linear distortion function

$$
\mathbb{K}(z, f):= \begin{cases}\frac{\|D f(z)\|^{2}}{J(z, f)} & \text { if } J(z, f)>0 \\ 1 & \text { otherwise. }\end{cases}
$$

The authors prove that for each mapping $f$ of finite distortion between annuli with the same boundary conditions as in Definition 7,

$$
\int_{A(q, 1)} \mathbb{K}\left(z, f_{0}\right) \frac{1}{|z|^{2}} \mathrm{~d} \mathcal{L}^{2}(z) \leq \int_{A(q, 1)} \mathbb{K}(z, f) \frac{1}{|z|^{2}} \mathrm{~d} \mathcal{L}^{2}(z),
$$

and the extremal map $f_{0}$ is unique up to rotation of the annuli. This is more general than the statement in Theorem 8 , in the sense that the mappings under consideration are not required to be of class $W_{l o c}^{1,2}$ with integrable distortion. On the other hand, we consider $\Psi(K(z, f))$ for an arbitrary convex non-decreasing function $\Psi$ instead of $\mathbb{K}(z, f)$. Note that the non-linear distortion can be written as

$$
\mathbb{K}(z, f)=\frac{1}{2}\left(K(z, f)+\frac{1}{K(z, f)}\right)=\Psi(K(z, f)),
$$

where $\Psi$ is the convex non-decreasing function $\Psi(x):=\frac{1}{2}(x+1 / x)$. The result for the mean $\mathbb{K}(z, f)$-distortion can thus be obtained as a particular case of Theorem 8 .

\section{Extremality of the spiral-stretch map. Proof of Theorem 6}

In the subsequent discussion, we always assume that $q \in(0,1), \theta \in[-\pi, \pi]$ and $k_{1}>0$ are arbitrary constants. Moreover, we assume in this section that $\theta \neq 0$ 
(and hence $k_{2} \neq 0$ ). The case $\theta=0$ can be obtained as a corollary to Theorem 8 , where the extremality of $f_{0}$ is proved even within a larger class of mappings than $\mathcal{F}$.

3.1 Strategy of the proof. The proof of Theorem 6 consists of several steps.

Step 1. In Section 3.2.1, we recall the definition of the modulus $M(\Gamma)$ of a curve family $\Gamma$ and cite the following modulus estimate for mappings of finite distortion, [20], which is one of the key tools in the proof:

$$
M(f(\Gamma)) \leq \int_{\Omega} K(z, f) \rho^{2}(z) \mathrm{d} \mathcal{L}^{2}(z) \quad \text { for all } \rho \in \operatorname{adm}(\Gamma) .
$$

In Section 3.2.2, we formulate sufficient conditions on a curve family $\Gamma_{0} \subset \Omega$ and a density $\rho_{0} \in \operatorname{adm}\left(\Gamma_{0}\right)$ to obtain equality in (1) for a quasiconformal map $f$ with constant distortion.

Step 2. In Section 3.3, our aim is to show that, for a fixed $N \in \mathbb{Z}$, the spiralstretch map $f_{N}$ is extremal in its subclass $\mathcal{F}_{N}$. Using the previous result, we identify a family $\Gamma_{0}$ of spirals and a density $\rho_{0}(z)=c /|z|$ such that

$$
\int_{\Omega} K\left(z, f_{N}\right) \rho_{0}^{2}(z) \mathrm{d} \mathcal{L}^{2}(z)=M\left(f_{N}\left(\Gamma_{0}\right)\right)
$$

with $\Omega=A(q, 1)$. To capture the class of mappings that are homotopic to $f_{N}$, we introduce a larger curve family $\Gamma$ that contains $\Gamma_{0}$ as a subfamily and satisfies

$$
\rho_{0} \in \operatorname{adm}(\Gamma)
$$

and

$$
M\left(f_{N}\left(\Gamma_{0}\right)\right) \leq M(f(\Gamma)) \text { for all } f \in \mathcal{F}_{N}
$$

The first two steps together yield the estimate

$$
\int_{\Omega} K\left(z, f_{N}\right) \rho_{0}^{2}(z) \mathrm{d} \mathcal{L}^{2}(z) \stackrel{(2)}{=} M\left(f_{N}\left(\Gamma_{0}\right)\right) \stackrel{(4)}{\leq} M(f(\Gamma)) \stackrel{(3),(1)}{\leq} \int_{\Omega} K(z, f) \rho_{0}^{2}(z) \mathrm{d} \mathcal{L}^{2}(z)
$$

for all $f \in \mathcal{F}_{\mathcal{N}}$. Since $\rho_{0}(z)$ is a constant multiple of $1 /|z|$, we obtain the statement of Theorem 5:

(5) $\quad \int_{A(q, 1)} K\left(z, f_{N}\right) \frac{1}{|z|^{2}} \mathrm{~d} \mathcal{L}^{2}(z) \leq \int_{A(q, 1)} K(z, f) \frac{1}{|z|^{2}} \mathrm{~d} \mathcal{L}^{2}(z) \quad$ for all $f \in \mathcal{F}_{N}$.

Step 3. Finally, in Section 3.4, we show that the spiral-stretch map $f_{0}$ is extremal among all admissible spiral-stretch maps

$$
K_{f_{0}} \leq K_{f_{N}} \text { for all } N \in \mathbb{Z}
$$


Hence

$\int_{A(q, 1)} K\left(z, f_{0}\right) \frac{1}{|z|^{2}} \mathrm{~d} \mathcal{L}^{2}(z) \stackrel{(6)}{\leq} \int_{A(q, 1)} K\left(z, f_{N}\right) \frac{1}{|z|^{2}} \mathrm{~d} \mathcal{L}^{2}(z) \stackrel{(5)}{\leq} \int_{A(q, 1)} K(z, f) \frac{1}{|z|^{2}} \mathrm{~d} \mathcal{L}^{2}(z)$

for all $f \in \mathcal{F}$. We exploit the monotonicity and the convexity of the given function $\Psi$ and conclude the proof of Theorem 6 by applying Jensen's inequality.

\subsection{Step 1: General approach to the minimisation problem by mod-}

ulus methods. It is the aim of this section to establish expressions for the modulus of the image of a curve family in terms of an integral average of the distortion. This is a general approach that can be used in specific cases to identify a candidate mapping as an extremal mapping of finite distortion in a given class.

3.2.1 Modulus of a curve family. General estimate. We start by recalling the definition of the modulus of a curve family; see, e.g., [19, 21] for more details.

A curve $\gamma$ in a domain $\Omega \subseteq \mathbb{C}$ is a continuous map $\gamma:[a, b] \rightarrow \Omega$. We denote both the map and its image by $\gamma$. A family of curves in $\Omega$ is denoted by $\Gamma$. A Borel function $\rho: \Omega \rightarrow[0, \infty]$ is called an admissible metric density for $\Gamma$ if

$$
\int_{\gamma} \rho(z)|\mathrm{d} z| \geq 1
$$

for all $\gamma \in \Gamma$ that are rectifiable. The set of admissible metric densities for $\Gamma$ is denoted by $\operatorname{adm}(\Gamma)$. For a Borel function $\rho$ and a rectifiable curve $\gamma:[a, b] \rightarrow \Omega$, the curve integral in (7) is given by $\int_{\gamma} \rho(z)|\mathrm{d} z|=\int_{0}^{l(\gamma)} \rho(\bar{\gamma}(s)) \mathrm{d} s$, where $\bar{\gamma}:[0, l(s)] \rightarrow \Omega$ is the arc-length parametrisation of $\gamma$. If $\gamma$ is absolutely continuous, $\int_{\gamma} \rho(z)|\mathrm{d} z|=\int_{a}^{b} \rho(\gamma(t))|\dot{\gamma}(t)| \mathrm{d} t$. The modulus of a curve family $\Gamma$ is defined as

$$
M(\Gamma):=\inf _{\rho \in \operatorname{adm}(\Gamma)} \int_{\Omega} \rho^{2}(z) \mathrm{d} \mathcal{L}^{2}(z) .
$$

Note that $M(\cdot)$ is an outer measure on the set of curves in $\Omega$ and that

$$
M(\Gamma)=M\left(\Gamma_{\text {rect }}\right)
$$

for the subfamily $\Gamma_{\text {rect }} \subseteq \Gamma$ of rectifiable curves.

To prove that a given mapping $f_{0} \in \mathcal{F}$ is a minimiser for the mean distortion among all mappings in the class $\mathcal{F}$, we should establish a lower bound on the mean distortion of mappings in $\mathcal{F}$ and show that this bound is achieved by our candidate map $f_{0}$. The essential modulus estimate is the following particular case of the estimate given in [20, Theorem 2.19] for $Q(x)$-quasiconformal mappings; see also [21, Theorem 4.1]. 
Theorem 9 ([20]). Let $\Omega$ be a domain in $\mathbb{C}$ and $\Gamma$ a curve family in $\Omega$. Let $f: \Omega \rightarrow \mathbb{C}$ be a homeomorphism onto its image and assume that $f$ belongs to the Sobolev class $W_{\text {loc }}^{1,2}(\Omega)$ and that it is of finite distortion with $K(\cdot, f) \in L_{\text {loc }}^{1}(\Omega)$. Then

$$
M(f(\Gamma)) \leq \int_{\Omega} K(z, f) \rho^{2}(z) \mathrm{d} \mathcal{L}^{2}(z) \quad \text { for all } \rho \in \operatorname{adm}(\Gamma) .
$$

Remark 10. A modulus estimate of this type was first established for quasiconformal mappings; see [1] and [17, p. 221].

3.2.2 Curve family for an optimal modulus estimate. In the next proposition, we formulate sufficient conditions for obtaining equality in (9). Equality is obtained if the family consists of curves that are tangential to directions of the largest shrinking of the candidate mapping $g$. In other words, $|\mathrm{d} g|=\left(\left|g_{z}\right|-\left|g_{\bar{z}}\right|\right)|\mathrm{d} z|$ should hold infinitesimally along the respective curves.

Theorem 11. Let $\Omega$ and $\Omega^{\prime}$ be two domains in $\mathbb{C}$ and let $g: \Omega \rightarrow \Omega^{\prime}$ be a quasiconformal map with $K(z, g) \equiv K_{g}$. Consider a family $\Gamma_{0}$ of absolutely continuous curves $\gamma$ in $\Omega$ with the following properties:

(i) for all $\gamma \in \Gamma_{0}$,

$$
\frac{g_{\bar{z}}(\gamma(s)) \dot{\bar{\gamma}}(s)}{g_{z}(\gamma(s)) \dot{\gamma}(s)}<0 \quad \text { for almost every } s ; \text { and }
$$

(ii) there exists $\rho_{0} \in \operatorname{adm}\left(\Gamma_{0}\right)$ such that $M\left(\Gamma_{0}\right)=\int_{\Omega} \rho_{0}^{2}(z) \mathrm{d} \mathcal{L}^{2}(z)$.

Then

$$
M\left(g\left(\Gamma_{0}\right)\right)=\int_{\Omega} K(z, g) \rho_{0}^{2}(z) \mathrm{d} \mathcal{L}^{2}(z) .
$$

Remark 12. Note that assumption (10) contains the requirement that the quotient on the left-hand side is real. Condition (10) can be written equivalently as

$$
g_{\bar{z}}(\gamma(s)) \dot{\bar{\gamma}}(s)=-\left|\mu_{g}(\gamma(s))\right| g_{z}(\gamma(s)) \dot{\gamma}(s)
$$

with $\dot{\gamma}(s) \neq 0$, for almost every $s$.

Remark 13. A statement of this type holds even if the existence of an extremal density $\rho_{0}$ is not required, as it follows from $[9, \mathrm{p} .182]$ that, for each curve family $\Gamma_{0}$, there exists a subfamily $\hat{\Gamma}_{0}$ with $M\left(\Gamma_{0} \backslash \hat{\Gamma}_{0}\right)=0$ and $\hat{\rho}_{0} \in \operatorname{adm}\left(\hat{\Gamma}_{0}\right)$ such that $M\left(\hat{\Gamma}_{0}\right)=\int_{\Omega} \hat{\rho}_{0}^{2}(z) \mathrm{d} \mathcal{L}^{2}(z)$. If the curves in $\Gamma_{0}$ satisfy the first assumption in the above theorem, one has $M\left(g\left(\hat{\Gamma}_{0}\right)\right)=\int_{\Omega} K(z, g) \hat{\rho}_{0}^{2}(z) \mathrm{d} \mathcal{L}^{2}(z)$. From the fact that $g$ is quasiconformal and $M\left(\Gamma_{0} \backslash \hat{\Gamma}_{0}\right)=0$, we conclude that the modulus $M\left(g\left(\Gamma_{0} \backslash \hat{\Gamma}_{0}\right)\right)$ vanishes; and thus $M\left(g\left(\Gamma_{0}\right)\right)=M\left(g\left(\hat{\Gamma}_{0}\right)\right)=\int_{\Omega} K(z, g) \hat{\rho}_{0}^{2}(z) \mathrm{d} \mathcal{L}^{2}(z)$. 
Proof of Theorem 11. Let $\Gamma_{0}$ satisfy assumptions (i) and assumptions (ii) of the theorem. Note that we may assume without loss of generality that the curves in $g\left(\Gamma_{0}\right)$ are absolutely continuous. The function $g$ belongs to $A C L^{2}$; that is, its coordinate functions are absolutely continuous on almost every line segment parallel to the coordinate axes, and the first partial derivatives exist almost everywhere and belong to $L_{l o c}^{2}$. It follows from Fuglede's theorem [9] (see, e.g., [27, p. 95] or [21, Theorem 2.7]) that $g$ is absolutely continuous on almost every curve in $\Gamma_{0}$, meaning that the sub-family of locally rectifiable curves in $\Gamma_{0}$ on which $g$ is absolutely continuous has the same modulus as $\Gamma_{0}$.

By (9), we have

$$
M\left(g\left(\Gamma_{0}\right)\right) \leq \int_{\Omega} K(z, g) \rho^{2}(z) \mathrm{d} \mathcal{L}^{2}(z) \quad \text { for all } \rho \in \operatorname{adm}\left(\Gamma_{0}\right)
$$

in particular,

$$
M\left(g\left(\Gamma_{0}\right)\right) \leq \int_{\Omega} K(z, g) \rho_{0}^{2}(z) \mathrm{d} \mathcal{L}^{2}(z) .
$$

We prove that the above inequalities are in fact equalities.

Since $J(z, g)>0$ a.e., we have $\left|g_{z}\right|-\left|g_{\bar{z}}\right|>0$ a.e. The push-forward density

$$
\rho^{\prime}(\zeta)=\frac{\rho\left(g^{-1}(\zeta)\right)}{\left|g_{z}\left(g^{-1}(\zeta)\right)\right|-\mid g_{\bar{z}}\left(g^{-1}(\zeta) \mid\right.}, \quad \zeta \in g(\Omega)
$$

can then be defined for every $\rho \in \operatorname{adm}\left(\Gamma_{0}\right)$. Note that the curves in $\Gamma_{0}$ and $g\left(\Gamma_{0}\right)$ are absolutely continuous. It can be shown (see, e.g., [1]) that, for all $\tilde{\gamma} \in g\left(\Gamma_{0}\right)$ and all $\rho \in \operatorname{adm}\left(\Gamma_{0}\right)$,

$$
\int_{\tilde{\gamma}} \rho^{\prime}(\zeta)|\mathrm{d} \zeta| \geq \int_{g^{-1} \circ \tilde{\gamma}} \rho(z)|\mathrm{d} z| \geq 1 .
$$

Hence $\rho^{\prime} \in \operatorname{adm}\left(g\left(\Gamma_{0}\right)\right)$ for $\rho \in \operatorname{adm}\left(\Gamma_{0}\right)$, i.e., the inclusion

$$
\left\{\rho^{\prime}: \rho \in \operatorname{adm}\left(\Gamma_{0}\right)\right\} \subseteq \operatorname{adm}\left(g\left(\Gamma_{0}\right)\right)
$$

always holds. By using (10), the reverse inclusion can be established as well.

Indeed, for $\tilde{\rho} \in \operatorname{adm}\left(g\left(\Gamma_{0}\right)\right)$ and $\gamma:[a, b] \rightarrow \Omega$ in $\Gamma_{0}$, we find

$$
\begin{aligned}
1 \leq \int_{g \circ \gamma} \tilde{\rho}(\zeta)|\mathrm{d} \zeta| & =\int_{a}^{b} \tilde{\rho}\left(g(\gamma(s))\left|g_{z}(\gamma(s)) \dot{\gamma}(s)+g_{\bar{z}}(\gamma(s)) \dot{\bar{\gamma}}(s)\right| \mathrm{d} s\right. \\
& \stackrel{(10)}{=} \int_{a}^{b} \tilde{\rho}(g(\gamma(s)))\left(\left|g_{z}(\gamma(s))\right|-\left|g_{\bar{z}}(\gamma(s))\right|\right)|\dot{\gamma}(s)| \mathrm{d} s \\
& =\int_{a}^{b} \rho(\gamma(s))|\dot{\gamma}(s)| \mathrm{d} s \\
& =\int_{\gamma} \rho(z)|\mathrm{d} z|,
\end{aligned}
$$


where we have set $\rho(z)=\tilde{\rho}(g(z))\left(\left|g_{z}(z)\right|-\left|g_{\bar{z}}(z)\right|\right)$ (then $\left.\rho^{\prime}(\zeta)=\tilde{\rho}(\zeta)\right)$. The above computation shows that $\rho \in \operatorname{adm}\left(\Gamma_{0}\right)$. We conclude that $\operatorname{adm}\left(g\left(\Gamma_{0}\right)\right) \subseteq\left\{\rho^{\prime}: \rho \in\right.$ $\left.\operatorname{adm}\left(\Gamma_{0}\right)\right\}$.

Altogether, we obtain, for a curve family $\Gamma_{0}$ that satisfies the conditions of the theorem,

$$
\operatorname{adm}\left(g\left(\Gamma_{0}\right)\right)=\left\{\rho^{\prime}: \rho \in \operatorname{adm}\left(\Gamma_{0}\right)\right\} .
$$

This implies

$$
\begin{aligned}
M\left(g\left(\Gamma_{0}\right)\right) & =\inf _{\tilde{\rho} \in \operatorname{adm}\left(g\left(\Gamma_{0}\right)\right)} \int_{g(\Omega)} \tilde{\rho}^{2}(\zeta) \mathrm{d} \mathcal{L}^{2}(\zeta) \\
& =\inf _{\rho \in \operatorname{adm}\left(\Gamma_{0}\right)} \int_{g(\Omega)}\left(\rho^{\prime}(\zeta)\right)^{2} \mathrm{~d} \mathcal{L}^{2}(\zeta) \\
& =\inf _{\rho \in \operatorname{adm}\left(\Gamma_{0}\right)} \int_{g(\Omega)}\left(\frac{\rho\left(g^{-1}(\zeta)\right)}{\left|g_{z}\left(g^{-1}(\zeta)\right)\right|-\left|g_{\bar{z}}\left(g^{-1}(\zeta)\right)\right|}\right)^{2} \mathrm{~d} \mathcal{L}^{2}(\zeta) .
\end{aligned}
$$

Since $g$ is of class $W_{l o c}^{1,1}$ and satisfies Lusin's condition $(N)$, we can apply the change of variables formula to $\zeta=g(z)$ for the measurable function

$$
u(\cdot)=\left(\frac{\rho\left(g^{-1}(\cdot)\right)}{\left|g_{z}\left(g^{-1}(\cdot)\right)\right|-\left|g_{\bar{z}}\left(g^{-1}(\cdot)\right)\right|}\right)^{2} \geq 0 .
$$

Hence

$$
\begin{aligned}
& \int_{g(\Omega)}\left(\frac{\rho\left(g^{-1}(\zeta)\right)}{\left|g_{z}\left(g^{-1}(\zeta)\right)\right|-\left|g_{\bar{z}}\left(g^{-1}(\zeta)\right)\right|}\right)^{2} \mathrm{~d} \mathcal{L}^{2}(\zeta) \\
& =\int_{\Omega} \frac{\rho^{2}(z)}{\left(\left|g_{z}(z)\right|-\left|g_{\bar{z}}(z)\right|\right)^{2}}|J(z, g)| \mathrm{d} \mathcal{L}^{2}(z) \\
& =\int_{\Omega} \frac{\rho^{2}(z)}{\left(\left|g_{z}(z)\right|-\left|g_{\bar{z}}(z)\right|\right)^{2}}\left(\left|g_{z}(z)\right|^{2}-\left|g_{\bar{z}}(z)\right|^{2}\right) \mathrm{d} \mathcal{L}^{2}(z) \\
& =\int_{\Omega} \frac{\left|g_{z}(z)\right|+\left|g_{\bar{z}}(z)\right|}{\left|g_{z}(z)\right|-\left|g_{\bar{z}}(z)\right|} \rho^{2}(z) \mathrm{d} \mathcal{L}^{2}(z)=\int_{\Omega} K(z, g) \rho^{2}(z) \mathrm{d} \mathcal{L}^{2}(z) .
\end{aligned}
$$

This yields

$$
\begin{aligned}
M\left(g\left(\Gamma_{0}\right)\right) & =\inf _{\rho \in \operatorname{adm}\left(\Gamma_{0}\right)} \int_{\Omega} K(z, g) \rho^{2}(z) \mathrm{d} \mathcal{L}^{2}(z)=K_{g} \cdot \inf _{\rho \in \operatorname{adm}\left(\Gamma_{0}\right)} \int_{\Omega} \rho^{2}(z) \mathrm{d} \mathcal{L}^{2}(z) \\
& =K_{g} \cdot M\left(\Gamma_{0}\right)=K_{g} \int_{\Omega} \rho_{0}^{2}(z) \mathrm{d} \mathcal{L}^{2}(z)=\int_{\Omega} K(z, g) \rho_{0}^{2}(z) \mathrm{d} \mathcal{L}^{2}(z)
\end{aligned}
$$


3.3 Step 2: Minimisation in a given homotopy class. We apply the general results from the previous section to the case that the domains $\Omega=A(q, 1)$ and $\Omega^{\prime}=A\left(q^{k_{1}}, 1\right)$ are annuli in the complex plane. Throughout this section, $N \in \mathbb{Z}$ is an arbitrary but fixed integer. We consider the spiral-stretch map

$$
f_{N}(z)=z|z|^{k_{1}-1} e^{\mathrm{i} k_{2} \log |z|}, \quad \text { with } k_{2}=k_{2}(N)=\frac{\theta+2 \pi N}{\log q} .
$$

Recall that we assume $k_{2} \neq 0$. The case $k_{2}=0$ is obtained as a corollary to Theorem 8.

In this section, we assign to each $f \in \mathcal{F}$ a winding number $N_{f}$ which measures the amount of twisting. The aim is to show that the spiral-stretch map $f_{N}$ is a minimiser for a weighted mean distortion in the class

$$
\mathcal{F}_{N}:=\left\{f \in \mathcal{F}: N_{f}=N\right\} .
$$

First, we use Theorem 11 to identify a curve family $\Gamma_{0}$ and a density $\rho_{0}$ for which equality holds in (9) with $g=f_{N}$ and $\Omega=A(q, 1)$, i.e.,

$$
\int_{A(q, 1)} K\left(z, f_{N}\right) \rho_{0}^{2}(z) \mathrm{d} \mathcal{L}^{2}(z)=M\left(f_{N}\left(\Gamma_{0}\right)\right) .
$$

Note that $f_{N}$ is a quasiconformal map with $K\left(z, f_{N}\right)=K_{f_{N}}$ for all $z \in A(q, 1)$. The aim is to find a family $\Gamma_{0, N}$ of absolutely continuous curves $\gamma:[q, 1] \rightarrow \overline{A(q, 1)}$ such that

$$
\frac{f_{N_{\bar{z}}}(\gamma(s)) \dot{\bar{\gamma}}(s)}{f_{N_{z}}(\gamma(s)) \dot{\gamma}(s)}<0 \text { for almost every } s .
$$

By inserting the explicit formulas

$$
f_{N_{z}}(z)=\frac{1}{2}\left(k_{1}+1+\mathrm{i} k_{2}\right)|z|^{k_{1}-1} e^{\mathrm{i} k_{2} \log |z|}
$$

and

$$
f_{N \bar{z}}(z)=\frac{1}{2}\left(k_{1}-1+\mathrm{i} k_{2}\right)\left(\frac{z}{\bar{z}}\right)|z|^{k_{1}-1} e^{\mathrm{i} k_{2} \log |z|}
$$

into inequality (12), one sees that $\Gamma_{0, N}$ can be taken to be a family of spirals of a certain curvature.

Definition 14. For $t \in \mathbb{R}$ and $\phi \in[0,2 \pi)$, define

$$
\gamma_{\phi}^{t}(s)=s e^{\mathrm{i}(t \log s+\phi)}, \quad s \in[q, 1] .
$$

We consider the curve family

$$
\Gamma_{0, N}=\left\{\gamma_{\phi}^{c_{N}}: \phi \in[0,2 \pi)\right\},
$$


where

(13)

$$
c_{N}=c\left(k_{1}, k_{2}\right)=\frac{-k_{1}^{2}-k_{2}^{2}+1-\sqrt{\left(k_{1}^{2}+k_{2}^{2}-1\right)^{2}+4 k_{2}^{2}}}{2 k_{2}}, \quad \text { and } k_{2}=k_{2}(N) \neq 0 \text {. }
$$

Lemma 15. The curve family $\Gamma_{0, N}$ has the following properties:

(i) $f_{N}$ is absolutely continuous on each $\gamma \in \Gamma_{0, N}$;

(ii) for all $\gamma \in \Gamma_{0, N}$,

$$
\frac{f_{N_{\bar{z}}}(\gamma(s)) \dot{\bar{\gamma}}(s)}{f_{N_{z}}(\gamma(s)) \dot{\gamma}(s)}<0 \quad \text { for all } s \in[q, 1]
$$

Proof. The first part of the statement is trivial. Any curve $\gamma$ in the family $\Gamma_{0, N}$ is of the form $\gamma=\gamma_{\phi}^{c_{N}}$ for some $\phi \in[0,2 \pi)$. Clearly, the map

$$
s \mapsto f_{N} \circ \gamma(s)=s^{k_{1}} e^{\mathrm{i}\left(\left(c_{N}+k_{2}\right) \log s+\phi\right)}, \quad s \in[q, 1]
$$

is absolutely continuous.

Inequality (14) can be verified by a direct computation. For $\gamma(s)=s e^{\mathrm{i}\left(c_{N} \log s+\phi\right)}$, one obtains

$$
\frac{f_{N_{\bar{z}}}(\gamma(s)) \dot{\bar{\gamma}}(s)}{f_{N_{z}}(\gamma(s)) \dot{\gamma}(s)}=\frac{1-\mathrm{i} c_{N}}{1+\mathrm{i} c_{N}} \frac{k_{1}-1+\mathrm{i} k_{2}}{k_{1}+1+\mathrm{i} k_{2}} .
$$

This last expression is a negative real number exactly whenever

$$
\left(1-\mathrm{i} c_{N}\right)^{2}\left(k_{1}-1+\mathrm{i} k_{2}\right)\left(k_{1}+1-\mathrm{i} k_{2}\right)<0 .
$$

By using the definition of $c_{N}$, we compute

$$
\begin{aligned}
& \left(1-\mathrm{i} c_{N}\right)^{2}\left(k_{1}-1+\mathrm{i} k_{2}\right)\left(k_{1}+1-\mathrm{i} k_{2}\right) \\
& \quad=-\frac{k_{1}^{2}+k_{2}^{2}-1+\sqrt{\left(k_{1}^{2}+k_{2}^{2}-1\right)^{2}+4 k_{2}^{2}}}{2 k_{2}^{2}}\left(\left(k_{1}^{2}+k_{2}^{2}-1\right)^{2}+4 k_{2}^{2}\right) .
\end{aligned}
$$

Note that this last expression is negative since

$$
k_{1}^{2}+k_{2}^{2}-1+\sqrt{\left(k_{1}^{2}+k_{2}^{2}-1\right)^{2}+4 k_{2}^{2}}>0 .
$$

Associated to the curve family $\Gamma_{0, N}$, we consider the following density.

Definition 16. Define the non-negative Borel function

$$
\rho_{0, N}(z)=\frac{1}{-\log q \cdot \sqrt{1+c_{N}^{2}}} \frac{1}{|z|}, \quad z \in A(q, 1) .
$$


Lemma 17. Let $\Gamma_{0, N}$ and $\rho_{0, N}$ be as above. Then

$$
\rho_{0, N} \in \operatorname{adm}\left(\Gamma_{0, N}\right) \quad \text { and } \quad M\left(\Gamma_{0, N}\right)=\int_{A(q, 1)} \rho_{0, N}^{2}(z) \mathrm{d} \mathcal{L}^{2}(z)=\frac{-2 \pi}{\left(1+c_{N}^{2}\right) \log q} .
$$

Proof. First, let us show that the density $\rho_{0, N}$ is admissible for $\Gamma_{0, N}$. To this end, observe that an absolutely continuous curve $\gamma:[q, 1] \rightarrow \overline{A(q, 1)}$ can be written as $\gamma(s)=|\gamma(s)| e^{\mathrm{i} \varphi(s)}$, where $\varphi:[q, 1] \rightarrow \mathbb{R}$ is an absolutely continuous angle function, which is unique up to the addition of a constant multiple of $2 \pi$. Using well-known properties of absolutely continuous functions, we find that

$$
\begin{aligned}
\int_{\gamma} \rho_{0, N}(z)|\mathrm{d} z| & =\frac{1}{-\log q \sqrt{1+c_{N}^{2}}} \int_{\gamma} \frac{1}{|z|}|\mathrm{d} z|=\frac{1}{-\log q \sqrt{1+c_{N}^{2}}} \int_{q}^{1} \frac{|\dot{\gamma}(s)|}{|\gamma(s)|} \mathrm{d} s \\
& \geq \frac{1}{-\log q \sqrt{1+c_{N}^{2}}}\left|\int_{q}^{1} \frac{\dot{\gamma}(s)}{\gamma(s)} \mathrm{d} s\right| \\
& =\frac{1}{-\log q \sqrt{1+c_{N}^{2}}}\left|\int_{q}^{1} \frac{\frac{d}{d s}|\gamma(s)|}{|\gamma(s)|}+\mathrm{i} \dot{\varphi}(s) \mathrm{d} s\right| .
\end{aligned}
$$

Hence, for every absolutely continuous curve $\gamma:[q, 1] \rightarrow \overline{A(q, 1)}$, we have

$$
\int_{\gamma} \rho_{0, N}(z)|\mathrm{d} z| \geq \frac{1}{-\log q \sqrt{1+c_{N}^{2}}}\left|\log \left(\frac{|\gamma(1)|}{|\gamma(q)|}\right)+\mathrm{i}(\varphi(1)-\varphi(q))\right| .
$$

In particular, $\int_{\gamma_{\phi}^{c_{N}}} \rho_{0, N}(z)|\mathrm{d} z| \geq 1$ for all $\phi \in[0,2 \pi)$, which proves that $\rho_{0, N}$ is admissible for $\Gamma_{0, N}$.

Next, we prove that the density $\rho_{0, N}$ is extremal for $\Gamma_{0, N}$. On the one hand, we have

$$
\begin{aligned}
M\left(\Gamma_{0, N}\right) & \leq \int_{A(q, 1)} \rho_{0, N}^{2}(z) \mathrm{d} \mathcal{L}^{2}(z) \\
& =\frac{1}{(-\log q)^{2}\left(1+c_{N}^{2}\right)} \int_{0}^{2 \pi} \int_{q}^{1} \frac{1}{r^{2}} r \mathrm{~d} r \mathrm{~d} \phi \\
& =\frac{-2 \pi}{\left(1+c_{N}^{2}\right) \log q} .
\end{aligned}
$$

On the other hand, let $\rho \in \operatorname{adm}\left(\Gamma_{0, N}\right)$ be an arbitrary admissible density for $\Gamma_{0, N}$ such that

$$
1 \leq \int_{\gamma_{\phi}^{c_{N}}} \rho(z)|\mathrm{d} z| \text { for all } \phi \in[0,2 \pi) .
$$

Then, for all $\phi \in[0,2 \pi)$,

$$
1 \leq \int_{q}^{1} \rho\left(s e^{\mathrm{i}\left(c_{N} \log s+\phi\right)}\right)\left|\left(1+\mathrm{i} c_{N}\right) e^{\mathrm{i}\left(c_{N} \log s+\phi\right)}\right| \mathrm{d} s=\int_{q}^{1} \rho\left(s e^{\mathrm{i}\left(c_{N} \log s+\phi\right)}\right) \sqrt{1+c_{N}^{2}} \mathrm{~d} s .
$$


Integrating both sides of the inequality with respect to $\phi$ from 0 to $2 \pi$ and applying the Cauchy-Schwarz inequality, Fubini's theorem, and the transformation formula, we obtain

$$
\begin{aligned}
2 \pi & \leq \sqrt{1+c_{N}^{2}} \int_{0}^{2 \pi} \int_{q}^{1} \rho\left(s e^{\mathrm{i}\left(c_{N} \log s+\phi\right)}\right) \sqrt{s} \frac{1}{\sqrt{s}} \mathrm{~d} s \mathrm{~d} \phi \\
& \leq \sqrt{1+c_{N}^{2}}\left(\int_{0}^{2 \pi} \int_{q}^{1}\left(\rho\left(s e^{\mathrm{i}\left(c_{N} \log s+\phi\right)}\right)\right)^{2} s \mathrm{~d} s \mathrm{~d} \phi\right)^{\frac{1}{2}}\left(\int_{0}^{2 \pi} \int_{q}^{1} \frac{1}{s} \mathrm{~d} s \mathrm{~d} \phi\right)^{\frac{1}{2}} \\
& =\sqrt{1+c_{N}^{2}}\left(\int_{A(q, 1)} \rho^{2} \circ f(z) \mathrm{d} \mathcal{L}^{2}(z)\right)^{\frac{1}{2}}(-2 \pi \log q)^{\frac{1}{2}}
\end{aligned}
$$

where $f(z):=z e^{i c_{N} \log |z|}$. Since $J(z, f)=1$ and $f(A(q, 1))=A(q, 1)$, applying the change of variables formula yields

$$
\begin{aligned}
2 \pi & \leq \sqrt{1+c_{N}^{2}}\left(\int_{A(q, 1)} \rho^{2}(f(z))|J(z, f)| \mathrm{d} \mathcal{L}^{2}(z)\right)^{\frac{1}{2}}(-2 \pi \log q)^{\frac{1}{2}} \\
& =\sqrt{1+c_{N}^{2}}\left(\int_{A(q, 1)} \rho^{2}(\zeta) \mathrm{d} \mathcal{L}^{2}(\zeta)\right)^{\frac{1}{2}}(-2 \pi \log q)^{\frac{1}{2}} .
\end{aligned}
$$

Thus,

$$
\int_{A(q, 1)} \rho^{2}(\zeta) \mathrm{d} \mathcal{L}^{2}(\zeta) \geq \frac{-2 \pi}{\left(1+c_{N}^{2}\right) \log q} \quad \text { for all } \rho \in \operatorname{adm}\left(\Gamma_{0, N}\right),
$$

and therefore

$$
M\left(\Gamma_{0, N}\right) \geq \frac{-2 \pi}{\left(1+c_{N}^{2}\right) \log q} .
$$

We conclude that

$$
M\left(\Gamma_{0, N}\right)=\int_{A(q, 1)} \rho_{0, N}^{2}(z) \mathrm{d} \mathcal{L}^{2}(z)=\frac{-2 \pi}{\left(1+c_{N}^{2}\right) \log q} .
$$

Proposition 18. Using the same notation as before, we have

$$
M\left(f_{N}\left(\Gamma_{0, N}\right)\right)=\frac{1}{(-\log q)^{2}\left(1+c_{N}^{2}\right)} \int_{A(q, 1)} K\left(z, f_{N}\right) \frac{1}{|z|^{2}} \mathrm{~d} \mathcal{L}^{2}(z) .
$$

Proof. This follows by applying Theorem 11 to $g=f_{N}, \Gamma_{0}=\Gamma_{0, N}$ with $c_{N}=c\left(k_{1}, k_{2}\right)$ and $\rho_{0}=\rho_{0, N}$. The necessary assumptions have been established in Lemmas 15 and 17. 
3.3.1 Modulus for homotopic curves and maps. Proposition 18 allows us to express the modulus of the family of spirals $f_{N}\left(\Gamma_{0, N}\right)$ with respect to the distortion of the spiral-stretch map $f_{N}$. Ideally, this value $M\left(f_{N}\left(\Gamma_{0, N}\right)\right)$ would serve as a lower bound for the modulus of the image family for an arbitrary $f \in \mathcal{F}$. However, the family $f\left(\Gamma_{0, N}\right)$ does not in general contain the spiral family $f_{N}\left(\Gamma_{0, N}\right)$. To remedy this, we enlarge the curve family $\Gamma_{0, N}$ in such a way that $\rho_{0, N}$ is still admissible for this larger family. Specifically, we consider all curves that are homotopic to arcs of spirals in $\Gamma_{0, N}$.

Recall that two curves, $\gamma_{0}:[q, 1] \rightarrow \overline{A(q, 1)}$ and $\gamma_{1}:[q, 1] \rightarrow \overline{A(q, 1)}$, with $\gamma_{0}(q)=\gamma_{1}(q)$ and $\gamma_{0}(1)=\gamma_{1}(1)$ are homotopic (with respect to the endpoints), $\gamma_{0} \simeq \gamma_{1}$, if there exists a continuous map

$$
H:[q, 1] \times[0,1] \rightarrow \overline{A(q, 1)}, \quad(s, t) \mapsto H(s, t),
$$

such that

$$
H(s, 0)=\gamma_{0}(s) \quad \text { and } H(s, 1)=\gamma_{1}(s) \text { for all } s \in[q, 1]
$$

and

$$
H(q, t)=\gamma_{0}(q)=\gamma_{1}(q) \text { and } H(1, t)=\gamma_{0}(1)=\gamma_{1}(1) \quad \text { for all } t \in[0,1]
$$

Definition 19. Set

$$
\Gamma_{N}=\left\{\gamma:[q, 1] \rightarrow \overline{A(q, 1)} \text { absolutely continuous, } \gamma \simeq \gamma_{\phi}^{c_{N}}, \phi \in[0,2 \pi)\right\}
$$

Lemma 20. The density $\rho_{0, N}$ is admissible for $\Gamma_{N}$.

Proof. Let $\gamma \in \Gamma_{N}$. By definition, such a curve is absolutely continuous and homotopic to $\gamma_{\phi}^{c_{N}}$, where $\phi \in[0,2 \pi)$ is such that $\gamma(1)=e^{\mathrm{i} \phi}$. Hence it can be written as $\gamma(s)=|\gamma(s)| e^{\mathrm{i} \varphi(s)}, s \in[q, 1]$, for an absolutely continuous function $\varphi:[q, 1] \rightarrow \mathbb{R}$ with

$$
\varphi(1)=\phi \quad \text { and } \quad \varphi(q)=\phi+c_{N} \log q \quad(\bmod 2 \pi) .
$$

Using the homotopy between $\gamma$ and $\gamma_{\phi}^{c_{N}}$, one can show that in fact $\varphi(q)=\phi+$ $c_{N} \log q$ and thus, by (15),

$$
\int_{\gamma} \rho_{0, N}(z)|\mathrm{d} z| \geq \frac{1}{-\log q \sqrt{1+c_{N}^{2}}}\left|-\log q-\mathrm{i} c_{N} \log q\right|=1 .
$$

As we have chosen $\gamma \in \Gamma_{N}$ arbitrarily, this shows that $\rho_{0, N}$ is admissible for $\Gamma_{N}$. 
It is now our goal to compare the modulus of $f_{N}\left(\Gamma_{0, N}\right)$ with that of $f\left(\Gamma_{N}\right)$. However, the value of $M\left(f\left(\Gamma_{N}\right)\right)$ is not the same for all $f$ since not all mappings in $\mathcal{F}$ twist the annulus by the same amount. To classify the mappings according to their rotation behaviour, we assign to each mapping $f$ in $\mathcal{F}$ a winding number $N_{f}$ which measures the amount of twisting. Here we use the boundary conditions for mappings in the class $\mathcal{F}$.

Definition 21. For $f \in \mathcal{F}$, let

$$
\begin{array}{r}
N_{f}=N_{f}(\phi, t)=\frac{\Im\left[\log \left(f \circ \gamma_{\phi}^{t}(q)\right)-\log \left(f \circ \gamma_{\phi}^{t}(1)\right)-\mathrm{i}(t \log q+\theta)\right]}{2 \pi \quad}, \\
(\phi, t) \in[0,2 \pi) \times \mathbb{R},
\end{array}
$$

where $\theta$ denotes the rotation angle from Definition 2 .

We can write $f \circ \gamma_{\phi}^{t}(s)=r(s) e^{\mathrm{i} \varphi(s)}$, where $r:[q, 1] \rightarrow\left[q^{k_{1}}, 1\right]$ is a continuous function with $r(q)=q^{k_{1}}, r(1)=1$, and $\varphi:[q, 1] \rightarrow \mathbb{R}$ is a continuous argument with

$$
\varphi(q)=\varphi(1)+t \log q+\theta \quad(\bmod 2 \pi) .
$$

We leave it to the reader to verify that

$$
(\phi, t) \mapsto N_{f}(\phi, t)=\frac{\varphi(q)-\varphi(1)-t \log q-\theta}{2 \pi}, \quad(\phi, t) \in[0,2 \pi) \times \mathbb{R}
$$

is a continuous integer-valued function and hence a constant.

The modulus $M\left(f\left(\Gamma_{N}\right)\right)$ can now be compared to $M\left(f_{N}\left(\Gamma_{0, N}\right)\right)$ for all $f$ in the subclass

$$
\mathcal{F}_{N}:=\left\{f \in \mathcal{F}: N_{f}=N\right\} .
$$

Essentially, we would like to show for a given $f \in \mathcal{F}_{N}$ that $f_{N}\left(\Gamma_{0, N}\right) \subseteq f\left(\Gamma_{N}\right)$, i.e., that any curve $\gamma^{\prime} \in f_{N}\left(\Gamma_{0, N}\right)$ can be written as $\gamma^{\prime}=f \circ \gamma$, where $\gamma$ belongs to $\Gamma_{N}$. This is not true, though, since the curve $\gamma=f^{-1} \circ \gamma^{\prime}$ need not be absolutely continuous. However, since a map in $W_{l o c}^{1,2}$ belongs to the space $A C L^{2}$, it is possible to apply Fuglede's theorem [9] and still conclude that $M\left(f_{N}\left(\Gamma_{0, N}\right)\right) \leq M\left(f\left(\Gamma_{N}\right)\right)$.

Proposition 22. With the same notation as before,

$$
M\left(f_{N}\left(\Gamma_{0, N}\right)\right) \leq M\left(f\left(\Gamma_{N}\right)\right) \text { for all } f \in \mathcal{F}_{N} .
$$

Proof. Let $\gamma^{\prime}$ be an arbitrary curve in $f_{N}\left(\Gamma_{0, N}\right)$. Then, there exists $\phi \in[0,2 \pi)$ such that $\gamma^{\prime}=f_{N} \circ \gamma_{\phi}^{c_{N}}$. Let $f$ be an arbitrary map in $\mathcal{F}_{N}$. Since $f$ is a homeomorphism, we may write

$$
f_{N} \circ \gamma_{\phi}^{c_{N}}=f \circ \gamma
$$


where

$$
\gamma:=f^{-1} \circ \gamma^{\prime}=f^{-1} \circ f_{N} \circ \gamma_{\phi}^{c_{N}} .
$$

By using the fact that $f$ belongs to $\mathcal{F}_{N}$, one can show in addition that

$$
f \circ \gamma=f_{N} \circ \gamma_{\phi}^{c_{N}} \simeq f \circ \gamma_{\phi}^{c_{N}},
$$

which proves that $\gamma$ is homotopic to $\gamma_{\phi}^{c_{N}}$. We leave the details to the reader.

In order to conclude that the curve $\gamma:=f^{-1} \circ \gamma^{\prime}$ lies in $\Gamma_{N}$ (and thus $\gamma^{\prime}$ is an element of $f\left(\Gamma_{N}\right)$ as desired), $\gamma$ must be absolutely continuous - which is generally not the case. This problem can be solved by applying Fuglede's theorem. Note that we have assumed our mappings to be homeomorphisms of integrable distortion; therefore $f^{-1} \in W_{l o c}^{1,2}$, which guarantees that Fuglede's theorem can be applied to the inverse map $f^{-1}$. Together with (8), this allows us to conclude that $M\left(f_{N}\left(\Gamma_{0, N}\right)\right)=M\left(\left\{\gamma^{\prime} \in f_{N}\left(\Gamma_{0, N}\right)\right.\right.$ rectifiable $: f^{-1}$ is absolutely continuous on $\left.\left.\gamma^{\prime}\right\}\right)$. As explained above, every rectifiable curve $\gamma^{\prime}$ in $f_{N}\left(\Gamma_{0, N}\right)$ on which $f^{-1}$ is absolutely continuous belongs to $f\left(\Gamma_{N}\right)$. Hence $M\left(\left\{\gamma^{\prime} \in f_{N}\left(\Gamma_{0, N}\right)\right.\right.$ rectifiable : $f^{-1}$ is absolutely continuous on $\left.\left.\gamma^{\prime}\right\}\right) \leq M\left(f\left(\Gamma_{N}\right)\right)$. This completes the proof.

3.3.2 Extremality in a subclass: Proof of Theorem 5. A combination of the previous results yields the extremality of the spiral-stretch map $f_{N}$ in its subclass.

Proof of Theorem 5. Let $N \in \mathbb{Z}$ be fixed. An arbitrary $f \in \mathcal{F}_{N}$ satisfies

$$
\begin{aligned}
\int_{A(q, 1)} K\left(z, f_{N}\right) \rho_{0, N}^{2}(z) \mathrm{d} \mathcal{L}^{2}(z) & \stackrel{(16)}{=} M\left(f_{N}\left(\Gamma_{0, N}\right)\right) \stackrel{(17)}{\leq} M\left(f\left(\Gamma_{N}\right)\right) \\
& \stackrel{(9)}{\leq} \int_{A(q, 1)} K(z, f) \rho_{0, N}^{2}(z) \mathrm{d} \mathcal{L}^{2}(z),
\end{aligned}
$$

where we have used in the last step the fact that $\rho_{0, N}$ is admissible for $\Gamma_{N}$. Since this density is a constant multiple of $1 /|z|$, the result follows.

3.4 Step 3: Comparing homotopy classes. Proof of Theorem 6. In order to derive Theorem 6 from Theorem 5, we show that the spiral-stretch map $f_{0}$ is minimal among all admissible spiral-stretch maps, i.e.,

$$
K_{f_{0}} \leq K_{f_{N}} \text { for all } N \in \mathbb{Z}
$$


where $f_{N}(z)=z|z|^{k_{1}-1} e^{\mathrm{i} k_{2}(N) \log |z|}$.

Note that the spiral-stretch map $f_{N}$ has constant distortion

$$
K_{f_{N}}=K\left(z, f_{N}\right)=\frac{\sqrt{\left(k_{1}+1\right)^{2}+k_{2}(N)^{2}}+\sqrt{\left(k_{1}-1\right)^{2}+k_{2}(N)^{2}}}{\sqrt{\left(k_{1}+1\right)^{2}+k_{2}(N)^{2}}-\sqrt{\left(k_{1}-1\right)^{2}+k_{2}(N)^{2}}} .
$$

To prove the extremality of $f_{0}$ among all $f_{N}, N \in \mathbb{Z}$, we consider the function

$$
x \mapsto \kappa(x):=\frac{\sqrt{\left(k_{1}+1\right)^{2}+x^{2}}+\sqrt{\left(k_{1}-1\right)^{2}+x^{2}}}{\sqrt{\left(k_{1}+1\right)^{2}+x^{2}}-\sqrt{\left(k_{1}-1\right)^{2}+x^{2}}} .
$$

Note that $\kappa\left(k_{2}(N)\right)=K_{f_{N}}$. A direct computation shows that $\kappa(x)$ is an even function and monotone increasing as $|x| \rightarrow \infty$. Since by assumption, $|\theta| \leq \pi$, it follows that $\left|k_{2}(0) \log q\right|=|\theta| \leq|\theta+2 \pi N|=\left|k_{2}(N) \log q\right|$, and thus $\left|k_{2}(0)\right| \leq\left|k_{2}(N)\right|$ for all $N \in \mathbb{Z}$. This implies $K_{f_{0}} \leq K_{f_{N}}$ for all $N \in \mathbb{Z}$.

Let $N \in \mathbb{Z}$ be arbitrary. By Theorem 5, the map $f_{N}$ is extremal in the subclass $\mathcal{F}_{N} \subset \mathcal{F}$. More precisely,

$$
\int_{A(q, 1)} K\left(z, f_{N}\right) \frac{1}{|z|^{2}} \mathrm{~d} \mathcal{L}^{2}(z) \leq \int_{A(q, 1)} K(z, f) \frac{1}{|z|^{2}} \mathrm{~d} \mathcal{L}^{2}(z) \quad \text { for all } f \in \mathcal{F}_{N} .
$$

Since the map $f_{0}$ is extremal among all the spiral-stretch maps $f_{N}, N \in \mathbb{Z}$, we may conclude that

$$
\int_{A(q, 1)} K\left(z, f_{0}\right) \frac{1}{|z|^{2}} \mathrm{~d} \mathcal{L}^{2}(z) \leq \int_{A(q, 1)} K\left(z, f_{N}\right) \frac{1}{|z|^{2}} \mathrm{~d} \mathcal{L}^{2}(z) \quad \text { for all } N \in \mathbb{Z} .
$$

A combination of (20) and (21) yields

$$
\int_{A(q, 1)} K\left(z, f_{0}\right) \frac{1}{|z|^{2}} \mathrm{~d} \mathcal{L}^{2}(z) \leq \int_{A(q, 1)} K(z, f) \frac{1}{|z|^{2}} \mathrm{~d} \mathcal{L}^{2}(z) \quad \text { for all } f \in \mathcal{F} .
$$

For every convex and non-decreasing function $\Psi$, by applying Jensen's inequality in (22), we obtain

$$
\begin{aligned}
\Psi\left(K\left(z, f_{0}\right)\right) & =\Psi\left(\frac{1}{\int_{A(q, 1)} \frac{1}{|z|^{2}} \mathrm{~d} \mathcal{L}^{2}(z)} \int_{A(q, 1)} K\left(z, f_{0}\right) \frac{1}{|z|^{2}} \mathrm{~d} \mathcal{L}^{2}(z)\right) \\
& \stackrel{(22)}{\leq} \Psi\left(\frac{1}{\int_{A(q, 1)} \frac{1}{|z|^{2}} \mathrm{~d} \mathcal{L}^{2}(z)} \int_{A(q, 1)} K(z, f) \frac{1}{|z|^{2}} \mathrm{~d} \mathcal{L}^{2}(z)\right) \\
& \leq \frac{1}{\int_{A(q, 1)} \frac{1}{|z|^{2}} \mathrm{~d} \mathcal{L}^{2}(z)} \int_{A(q, 1)} \Psi(K(z, f)) \frac{1}{|z|^{2}} \mathrm{~d} \mathcal{L}^{2}(z),
\end{aligned}
$$

which yields the result

$$
\int_{A(q, 1)} \Psi\left(K\left(z, f_{0}\right)\right) \frac{1}{|z|^{2}} \mathrm{~d} \mathcal{L}^{2}(z) \leq \int_{A(q, 1)} \Psi(K(z, f)) \frac{1}{|z|^{2}} \mathrm{~d} \mathcal{L}^{2}(z) \quad \text { for all } f \in \mathcal{F} .
$$




\section{Extremality of the radial stretching. Proof of Theorem 8}

In the subsequent discussion, we assume that $q \in(0,1)$ and $k_{1}>0$ are arbitrary constants.

Proof of Theorem 8. The arguments used in this proof are similar to those used in the proof of Theorem 6. Therefore, we only sketch the proof, illustrating how to apply the modulus method based on Theorem 9, and leave the details to the interested reader.

The crucial point in the proof is to find an appropriate curve family $\Gamma_{0}$ and a metric density $\rho_{0}$ that is extremal for $\Gamma_{0}$ such that the assumptions of Theorem 11 hold with

$$
g(z)=f_{0}(z)=z|z|^{k_{1}-1}
$$

The geometric interpretation of $\Gamma_{0}$ is that it contains curves that are tangential to directions of largest shrinking (least stretching) of $f_{0}$. We consider two cases; $k_{1}>1$ and $k_{1}<1$.

Case $k_{1}>1$. We employ the curve family

$$
\Gamma_{0}=\left\{\gamma_{r}: r \in(q, 1)\right\}
$$

which contains the circles

$$
\gamma_{r}(s)=r e^{\mathrm{i} s}, \quad s \in[0,2 \pi] .
$$

The associated extremal density is given by

$$
\rho_{0}(z)=\frac{1}{2 \pi|z|}, \quad z \in A(q, 1) .
$$

One verifies easily that

$$
M\left(\Gamma_{0}\right)=\int_{A(q, 1)} \rho_{0}^{2}(z) \mathrm{d} \mathcal{L}^{2}(z)=\frac{-\log q}{2 \pi} .
$$

Since $\Gamma_{0}, \rho_{0}$ and $f_{0}$ satisfy the assumptions in Theorem 11, we conclude that

$$
\int_{A(q, 1)} K\left(z, f_{0}\right) \frac{1}{|z|^{2}} \mathrm{~d} \mathcal{L}^{2}(z)=(2 \pi)^{2} M\left(f_{0}\left(\Gamma_{0}\right)\right) .
$$

Again, the idea is to enlarge the curve family $\Gamma_{0}$ so that $\Gamma \supseteq \Gamma_{0}$ satisfies

(i) $\rho_{0} \in \operatorname{adm}(\Gamma)$ and

(ii) $M\left(f_{0}\left(\Gamma_{0}\right)\right) \leq M(f(\Gamma))$ for all $f \in \mathcal{G}$. 
Prior to the definition of this curve family, we recall the concept of the winding number of a closed curve. For every continuous curve $\gamma:[a, b] \rightarrow \mathbb{C} \backslash\{0\}$, a continuous function ("polar angle function") $\varphi:[a, b] \rightarrow \mathbb{R}$ can be defined such that

$$
\gamma(t)=|\gamma(t)| e^{\mathrm{i} \varphi(t)} .
$$

The difference $\varphi(b)-\varphi(a)$ is independent of the choice of the angle function and it can be used to define the winding number about the origin

$$
n(\gamma, 0)=\frac{1}{2 \pi}(\varphi(b)-\varphi(a)) \in \mathbb{Z} .
$$

If $\gamma$ is a closed piecewise $\mathcal{C}^{1}$-curve in $\mathcal{C} \backslash\{0\}$, then

$$
n(\gamma, 0)=\frac{1}{2 \pi \mathrm{i}} \int_{\gamma} \frac{1}{z} \mathrm{~d} z
$$

We consider the curve family

$$
\Gamma=\{\gamma:[0,2 \pi] \rightarrow A(q, 1) \text { absolutely continuous, } \gamma(0)=\gamma(2 \pi), n(\gamma, 0) \neq 0\}
$$

The density $\rho_{0}$ is admissible for this larger family, too, i.e.,

$$
\int_{\gamma} \rho_{0}(z)|\mathrm{d} z|=\frac{1}{2 \pi} \int_{0}^{2 \pi} \frac{|\dot{\gamma}(s)|}{|\gamma(s)|} \mathrm{d} s \geq \frac{1}{2 \pi}\left|\int_{0}^{2 \pi} \frac{\dot{\gamma}(s)}{\gamma(s)} \mathrm{d} s\right|=|n(\gamma, 0)| \geq 1 .
$$

By Fuglede's theorem and (8), we have $M\left(f_{0}\left(\Gamma_{0}\right)\right)=M\left(\left\{\gamma^{\prime} \in f_{0}\left(\Gamma_{0}\right): \gamma^{\prime}\right.\right.$ rectifiable, $f^{-1}$ absolutely continuous on $\left.\left.\gamma^{\prime}\right\}\right)$.

For a given map $f \in \mathcal{G}$, each such curve $\gamma^{\prime} \in f_{0}\left(\Gamma_{0}\right)$ can be written as $\gamma^{\prime}=f \circ \gamma$, where $\gamma=f^{-1} \circ \gamma^{\prime}$ is absolutely continuous by assumption and $n(\gamma, 0) \neq 0$ since $f$ is a homeomorphism between the two annuli. Hence $\gamma \in \Gamma$ and $\gamma^{\prime} \in f(\Gamma)$. We conclude that

$$
M\left(f_{0}\left(\Gamma_{0}\right)\right) \leq M(f(\Gamma)) .
$$

This yields the desired estimate

$$
\begin{aligned}
\int_{A(q, 1)} K\left(z, f_{0}\right) \frac{1}{|z|^{2}} \mathrm{~d} \mathcal{L}^{2}(z) & \stackrel{(23)}{=}(2 \pi)^{2} M\left(f_{0}\left(\Gamma_{0}\right)\right) \\
& \stackrel{(24)}{\leq}(2 \pi)^{2} M(f(\Gamma)) \\
& \stackrel{(9)}{\leq} \int_{A(q, 1)} K(z, f) \frac{1}{|z|^{2}} \mathrm{~d} \mathcal{L}^{2}(z) .
\end{aligned}
$$


Case $k_{1}<1$. we employ the family

$$
\Gamma_{0}=\left\{\gamma_{\phi}: \phi \in[0,2 \pi)\right\}
$$

of radial segments

$$
\gamma_{\phi}(s)=s e^{\mathrm{i} \phi}, \quad s \in[q, 1] .
$$

The associated extremal density is given by

$$
\rho_{0}(z)=\frac{1}{-\log q} \frac{1}{|z|} .
$$

Notice that these are exactly the same curve family and density as in Definitions 14 and 16 , respectively, for $c_{N}=0$. The reasoning of the proof of the extremal property of $f_{0}$ is therefore very similar to that in Section 3. To obtain extremality not only in the class $\mathcal{F}$ of mappings with pointwise boundary conditions but also in the larger class $\mathcal{G}$, one has to consider not just curves homotopic to $\gamma_{\phi}, \phi \in[0,2 \pi)$, but instead work with the larger family $\Gamma$ of all absolutely continuous curves that connect the two boundary components of $A(q, 1)$. One can show that

$$
M\left(f_{0}\left(\Gamma_{0}\right)\right) \leq M(f(\Gamma)) \text { for all } f \in \mathcal{G}
$$

and proceed as before.

Remark 23. An important question related to the minimisation of the distortion concerns the uniqueness of the solution. Even in the case of quasiconformal mappings and maximal distortion, the solution of the extremal problem for given boundary values, in general, is not unique, as can be seen by Strebel's famous chimney example [23]. But there are uniqueness results for quasiconformal mappings having a Beltrami coefficient of a particular form in a certain subclass [24].

Less is known about the uniqueness of extremal mappings of finite distortion. In [5], the authors considered a variant of the classical Grötzsch extremal problem for finite distortion mappings between rectangles. They proved the existence of a unique minimiser for an integrated non-linear distortion function $\mathbb{K}(z, f)$. However, the uniqueness is lost if one replaces the non-linear distortion by the usual linear distortion $K(z, f)$.

We stress here the fact that minimising the maximal distortion and minimising the mean distortion are indeed two problems of a different nature. As before, we fix constants $k_{1}>1$ and $q \in(0,1)$ and let $\mathcal{G}$ be as in Theorem 8 (see Definition 7). It is a classical result that the radial stretching

$$
f_{0}: A(q, 1) \rightarrow A\left(q^{k_{1}}, 1\right), \quad z \mapsto z|z|^{k_{1}-1}
$$


is the unique solution for the minimisation of the maximal distortion

$$
f \mapsto K_{f}
$$

within the subclass of quasiconformal maps in the class $\mathcal{G}$. According to Theorem 8 , it is also a minimiser for the mean distortion functional

$$
f \mapsto \int_{A(q, 1)} \Psi(K(z, f)) \frac{1}{|z|^{2}} \mathrm{~d} \mathcal{L}^{2}(z)
$$

in the class $\mathcal{G}$. A direct computation yields

$$
K\left(z, f_{0}\right)=K_{f_{0}}=k_{1} \quad \text { and } \quad \int_{A(q, 1)} \Psi\left(K\left(z, f_{0}\right)\right) \frac{1}{|z|^{2}} \mathrm{~d} \mathcal{L}^{2}(z)=-2 \pi \cdot \log q \cdot \Psi\left(k_{1}\right) .
$$

It is possible to construct another mapping $f_{1} \in \mathcal{G}$ which has a larger maximal distortion but the same weighted mean distortion as $f_{0}$ - at least for a specific choice of $\Psi$. In fact, there are infinitely many such mappings that are equal to the identity map on an outer ring and stretch an inner ring by a larger amount than $f_{0}$. These maps clearly increase the maximal distortion, but maps can be found that preserve the mean distortion, as we are integrating a larger distortion over a smaller area. More precisely, we consider

$$
f_{1}(z)= \begin{cases}z\left(\frac{|z|}{\tilde{q}}\right)^{\tilde{k}-1}, & z \in A(q, \tilde{q}), \\ z, & z \in A(\tilde{q}, 1) .\end{cases}
$$

Here, $\tilde{k}>k$ and $\tilde{q} \in(q, 1)$ are chosen so that $f_{1} \in \mathcal{G}$, i.e.,

$$
\left|f_{1}(z)\right|=|z|\left(\frac{|z|}{\tilde{q}}\right)^{\tilde{k}-1}=q\left(\frac{q}{\tilde{q}}\right)^{\tilde{k}-1} \stackrel{!}{=} q^{k_{1}} \quad \text { for }|z|=q .
$$

This is equivalent to

$$
\tilde{k}(\log q-\log \tilde{q})+\log \tilde{q}=k_{1} \log q
$$

We compute

$$
\mu_{f_{1}}(z)=\left\{\begin{array}{ll}
\frac{\tilde{k}-1}{\tilde{k}+1} \frac{z}{\bar{z}}, & z \in A(q, \tilde{q}), \\
0, & z \in A(\tilde{q}, 1),
\end{array} \quad K\left(z, f_{1}\right)=\left\{\begin{array}{ll}
\tilde{k}, & z \in A(q, \tilde{q}), \\
1, & z \in A(\tilde{q}, 1),
\end{array} \quad K_{f_{1}}=\tilde{k},\right.\right.
$$

and

$$
\int_{A(q, 1)} \Psi\left(K\left(z, f_{1}\right)\right) \frac{1}{|z|^{2}} \mathrm{~d} \mathcal{L}^{2}(z)=2 \pi(\Psi(\tilde{k})(\log \tilde{q}-\log q)-\log \tilde{q}) .
$$

This shows that

$$
K_{f_{0}}<K_{f_{1}} .
$$


Yet the mean distortions are equal for $\Psi(t)=t$, i.e.,

$$
\int_{A(q, 1)} K\left(z, f_{0}\right) \frac{1}{|z|^{2}} \mathrm{~d} \mathcal{L}^{2}(z) \stackrel{(25)}{=} \int_{A(q, 1)} K\left(z, f_{1}\right) \frac{1}{|z|^{2}} \mathrm{~d} \mathcal{L}^{2}(z) .
$$

On the other hand, if $\Psi$ is strictly convex, we have

$$
\Psi\left(k_{1}\right) \stackrel{(25)}{=} \Psi\left(\tilde{k}\left(1-\frac{\log \tilde{q}}{\log q}\right)+\frac{\log \tilde{q}}{\log q}\right)<\left(1-\frac{\log \tilde{q}}{\log q}\right) \Psi(\tilde{k})+\frac{\log \tilde{q}}{\log q} \Psi(1) ;
$$

and thus

$$
\int_{A(q, 1)} \Psi\left(K\left(z, f_{0}\right)\right) \frac{1}{|z|^{2}} \mathrm{~d} \mathcal{L}^{2}(z)<\int_{A(q, 1)} \Psi\left(K\left(z, f_{1}\right)\right) \frac{1}{|z|^{2}} \mathrm{~d} \mathcal{L}^{2}(z) .
$$

\section{Final remarks}

The modulus method by curve families used in this paper can be applied to other minimisation problems for a weighted mean distortion with fixed boundary values and a conjectured extremal mapping.

Remark 24. Bishop, Gutlyanskǐr, Martio and Vuorinen studied in [6] modulus estimates for quasiconformal mappings on domains in $\mathbb{R}^{n}, n \geq 2$. We believe that an analogue of our approach can be used to treat higher dimensional extremal problems of mappings of finite distortion, too.

As explained before, our method can be used to identify a quasiconformal map $f_{0}$ as a minimiser of the mean distortion functional in the class of finite distortion maps. In all the examples above, this conjectured extremal $f_{0}$ has a Beltrami coefficient of the form

$$
\mu(z)=k\left|\varphi_{0}\right| / \varphi_{0}, \quad \text { where } 0 \leq k<1
$$

and $\varphi_{0}$ is a holomorphic function on the annulus, i.e., a Teichmüller differential. Moreover, $\mu$ is related to the extremal density $\rho_{0}$ associated to $f_{0}$ and the curve family $\Gamma_{0}$ as in Theorem 11, via the identity $\rho_{0}(z)=\left|\varphi_{0}(z)\right|^{\frac{1}{2}}$. In the classical Teichmüller theory, quasiconformal maps with a Beltrami coefficient of the form (26) are shown to be unique extremals for their boundary values [25, 26]; see, e.g., $[24,10]$. The proof of the uniqueness is based on the length-area principle, using the trajectories of the so-called quadratic differential $\varphi_{0}$. Horizontal trajectories of $\varphi_{0}$ are lines of largest stretching, lines along which $\varphi_{0}(z) \mathrm{d} z^{2}>0$, whereas lines of largest shrinking are called vertical trajectories and are defined by the condition $\varphi_{0}(z) \mathrm{d} z^{2}<0$. 
Remark 25. We find that if the Beltrami coefficient of a quasiconformal map $g$ on a domain $\Omega$ in $\mathbb{C}$ is a Teichmüller differential (i.e., it has the form (26)), then the family $\Gamma_{0}$ of curves satisfying condition (10) in Theorem 11 consists exactly of the vertical trajectories of $\varphi_{0}$. Notice further that a Teichmüller map obviously always has constant distortion $K(z, g)=K_{g}$, hence is of the form required in Theorem 11.

Although related to the classical quasiconformal theory in this sense, the problems under consideration in this paper are of a different nature, as the goal is to minimise the mean distortion and the method yields extremal mappings in the larger class of finite distortion maps.

Remark 26. Observee that minimisers obtained by our method are quasiconformal with constant distortion, as Theorem 11 can only be applied if the conjectured extremal is of this form. As shown in [5, Theorem 11.27], the minimiser of the mean distortion

$$
f \mapsto \int_{\Omega} \mathbb{K}(z, f) \mathrm{d} \mathcal{L}^{2}(z)
$$

with fixed boundary data $f_{0}$ and $\Omega$ the unit disk is seldom quasiconformal. In fact, the minimiser is quasiconformal only if $f_{0}$ is bi-Lipschitz. It would be an interesting subject for further research to see if an analogous result holds for the weighted mean distortion $f \mapsto \int_{\Omega} \Psi(K(z, f)) \mathrm{d} \mu(z)$ for a convex non-decreasing function $\Psi$ and $\mathrm{d} \mu(z)=\rho_{0}^{2}(z) \mathrm{d} \mathcal{L}^{2}(z)$. Should this be the case, it will be of particular importance to develop further techniques for the identification of non-quasiconformal extremal maps of finite distortion.

Remark 27. We note that the minimisation of the integral mean (27) is a problem of a different nature from the minimisation of the weighted mean distortion functional

$$
f \mapsto \int_{\Omega} \Psi(K(z, f)) \mathrm{d} \mu(z),
$$

where $\mathrm{d} \mu(z)=\rho_{0}^{2}(z) \mathrm{d} \mathcal{L}^{2}(z)$ with the density $\rho_{0}$ coming from the conditions in Theorem 11.

It has been shown recently in [3] that the infimum of (27) in the class of finite distortion homeomorphisms between two annuli $A$ and $A^{\prime}$ that map the inner and the outer boundary of $A$ to the inner and outer boundary of $A^{\prime}$, respectively, is attained only when the image annulus $A^{\prime}$ is not too fat compared to $A$. The condition on the moduli can be explicitly described by the so-called Nitsche bound appearing in Nitsche's conjecture [22]. This result is in strong contrast to the analogous 
minimisation problem of the weighted mean distortion where one can always identify a minimiser. It would be interesting to study further the relation between the choice of the density and the existence of a minimiser for the associated mean distortion functional.

Acknowledgements. We thank Melkana Brakalova for helpful discussions on the subject of this paper. Also, we thank the referee for valuable comments.

\section{REFERENCES}

[1] L. V. Ahlfors, Lectures on Quasiconformal Mappings, D. Van Nostrand Co., 1966.

[2] K. Astala, Area distortion of quasiconformal mappings, Acta Math. 173 (1994), 37-60.

[3] K. Astala, T. Iwaniec and G. Martin, Deformations of Annuli with Smallest Mean Distortion, Arch. Ration. Mech. Anal. 195 (2009), 899-921.

[4] K. Astala, T. Iwaniec and G. Martin, Elliptic Partial Differential Equations and Quasiconformal Mappings in the Plane, Princeton University Press, Princeton, NJ, 2009.

[5] K. Astala, T. Iwaniec, G. J. Martin and J. Onninen, Extremal mappings of finite distortion, Proc. London Math. Soc. (3) 91 (2005), 655-702.

[6] C. J. Bishop, V. Ya Gutlyanskiı̌, O. Martio and M. Vuorinen, On conformal dilatation in space, Int. J. Math. Math. Sci. 2003 (2003), 1397-1420.

[7] M. A. Brakalova, Sufficient and necessary conditions for Conformality at a point I. Geometric viewpoint, Complex Var. Elliptic Equations 54 (2010), 137-155.

[8] M. A. Brakalova, Sufficient and necessary conditions for conformality II. Analytic viewpoint, Ann. Acad. Sci. Fenn. 35 (2010), 235-254.

[9] B. Fuglede, Extremal length and functional completion, Acta Math. 98 (1957), 171-219.

[10] F. P. Gardiner and N. Lakic, Quasiconformal Teichmüller Theory, American Mathematical Society, Providence, RI, 2000.

[11] F. W. Gehring, The $L^{p}$-integrability of the partial derivatives of a quasiconformal mapping, Acta Math. 130 (1973), 265-277.

[12] V. Gutlyanskiı̌ and O. Martio, Rotation estimates and spirals, Conform. Geom. Dyn. 5 (2001), 6-20 (electronic).

[13] T. Iwaniec, P. Koskela and J. Onninen, Mappings of finite distortion: monotonicity and continuity, Invent. Math. 144 (2001), 507-531.

[14] F. John, Rotation and strain, Comm. Pure Appl. Math. 14 (1961), 391-413.

[15] J. Kauhanen, P. Koskela and J. Malý, Mappings of finite distortion: condition N, Michigan Math. J. 49 (2001), 169-181.

[16] J. Kauhanen, P. Koskela and J. Malý, Mappings of finite distortion: discreteness and openness, Arch. Ration. Mech. Anal. 160 (2001), 135-151.

[17] O. Lehto and K. I. Virtanen, Quasiconformal Mappings in the Plane, second edn., SpringerVerlag, New York, 1973.

[18] G. J. Martin, The Teichmüller problem for mean distortion, Ann. Acad. Sci. Fenn. Math. 34 (2009), 233-247.

[19] O. Martio, Nonlinear potential theory in metric spaces, in Topics in Mathematical Analysis, World Scientific, Hackensack, NJ, 2008, pp. 29-60.

[20] O. Martio, V. Ryazanov, U. Srebro and E. Yakubov, On Q-homeomorphisms, Ann. Acad. Sci. Fenn. Math. 30 (2005), 49-69. 
[21] O. Martio, V. Ryazanov, U. Srebro and E. Yakubov, Moduli in Modern Mapping Theory, Springer, New York, 2009.

[22] J. C. C. Nitsche, On the module of doubly-connected regions under harmonic mappings, Amer. Math. Monthly 69 (1962), 781-782.

[23] K. Strebel, Zur Frage der Eindeutigkeit extremaler quasikonformer Abbildungen des Einheitskreises, Comment. Math. Helv. 36 (1961/1962), 306-323.

[24] K. Strebel, Extremal quasiconformal mappings, Results Math. 10 (1986), 168-210.

[25] O. Teichmüller, Extremale quasikonforme Abbildungen und quadratische Differentiale, Abh. Preuss. Akad. Wiss. Math.-Nat. K1. 1939 (1940), no. 22.

[26] O. Teichmüller, Bestimmung der extremalen quasikonformen Abbildung bei geschlossenen orientierten Riemannschen Flächen, Abh. Preuss. Akad. Wiss. Math.-Nat. K1. 1943 (1943), no. 4.

[27] J. Väisälä, Lectures on n-Dimensional Quasiconformal Mappings, Lecture Notes in Mathematics 229, Springer-Verlag, Berlin, 1971.
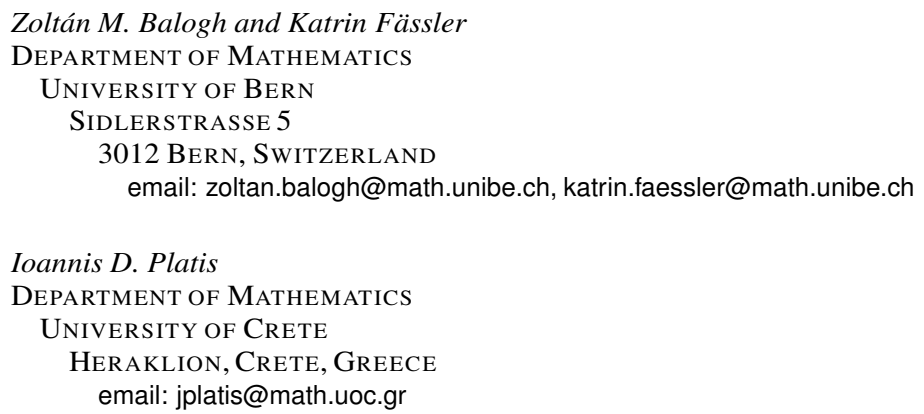

(Received November 28, 2009) 